\title{
Obturation Techniques and sealers used in Jodhpur City - A survey
}

\author{
${ }^{1}$ chinmayvyas, ${ }^{2}$ Dr.Anikaagarwal, ${ }^{3}$ Dr Sumitakaswan, ${ }^{4}$ Dr.Pankajscancheti. \\ ${ }^{I}$ PG Student, Department Of Conservative Dentistry And Endodontics, Jodhpur Dental College General \\ Hospital, Jodhpur Rajasthan, India. \\ ${ }^{2}$ Reader Lecturer, Department Of Conservative Dentistry And Endodontics, Jodhpur Dental College General \\ Hospital, Jodhpur Rajasthan, India. \\ ${ }^{3}$ Professor And Head, Department Of Conservative Dentistry And Endodontics, Jodhpur Dental College \\ General Hospital, Jodhpur Rajasthan, India. \\ ${ }^{4}$ Senior Lecturer, Department Of Conservative Dentistry And Endodontics, Jodhpur Dental College General \\ Hospital, Jodhpur Rajasthan, India.
}

\begin{abstract}
The importance of dental health has increased with the increased average age of patient, awareness regarding dental status and more likeliness of retaining natural teeth for a longer life span. With the increasing demand of retaining teeth, tooth saving procedures like root canal therapy has been more popular and number of this procedure has increased manifold in last 10 years.

Root canal therapy involves various steps among which a three dimensional filling is the last and crucial step of this procedure. The goal of root canal filling is to create a three-dimensional sealing in order to prevent the recurrence of bacterial infection. Several obturation techniques are used for the filling of root canals: the lateral condensation, Tagger's hybrid, vertical compaction, and thermoplasticized techniques.

This survey will evaluate various obturation techniques and sealers used for root canal therapy in the Jodhpur city. All the practioners are being included in the survey. The results may help in generating awareness and modification in techniques of obturation and the choice of sealer to enhance treatment outcome.
\end{abstract}

\section{Introduction}

With the increasing demand of retaining teeth, tooth saving procedures like root canal therapy has been more popular and number of this procedure has increased in last decade. Root canal therapy involves various steps among which a three dimensional filling is the last and crucial step of this procedure. The goal of root canal filling is to create a three dimensionalintracanal seal in order to prevent the recurrence of bacterial infection. Several obturation techniques are used for the filling of root canals: the lateral condensation, vertical compaction, thermoplasticized technique, sectional obturation and many others. Each of these techniques has their own indications and contraindications. It is important for a clinician to know about each of these techniques and their application in specific cases for long term endodontic success.

Aim of the Survey - To evaluate various obturation techniques and most commonly used sealer for root canal therapy by local practitioners in Jodhpur, Rajasthan.

Survey Form -

Jodhpur Dental College General Hospital Department Of Conservative Dentistry And Endodontics

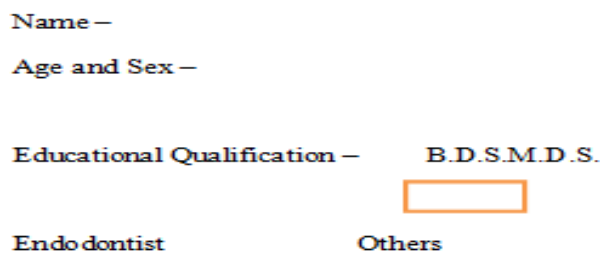

Practicing since Years, Months.

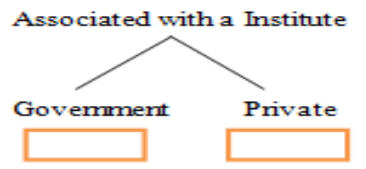

Private Practice Only 
Is Root Canal Treatment perfomed in your clinical practice?

Yes

No

Is single visit Root Canal Treatment performed in your clinical practice?

Yes

No

Order of steps followed in multiple visit Root Canal Treatment

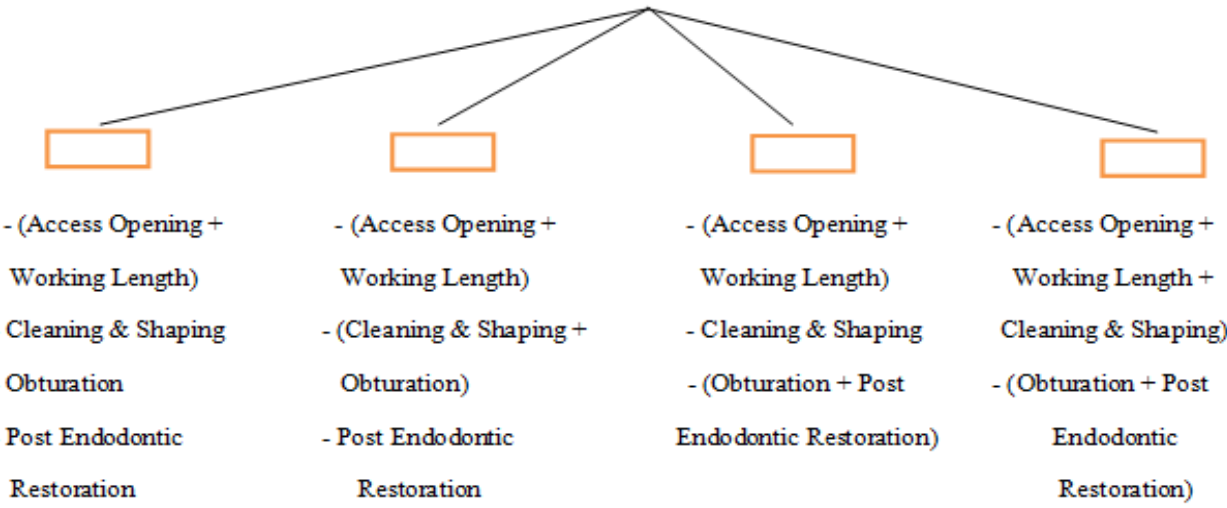

Restoration

Restoration

days

Obturation technique being followed
A) Vertical compaction
B) Lateral compaction
C) Segmental method
D) Combination method

1) Multiple cones

2) Single greater taper cone

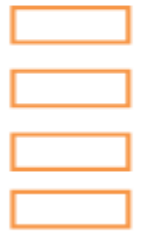

Sealer used for Obturation (Product name, can be one or more)

Method of application of Sealer

Questionnaire included in survey - Years of practice,time of obturation (days) after cleaning and shaping, Number of steps followed in multiple visit root canal treatment, obturation technique being followed,sealer of choice, method of application of sealer into the canal.

Results - Mean age of the practicing dentists in Jodhpur was 35.8 years with a standard deviation of \pm 9.06 and range of 24 to 73 years. Years of Endodontic Service - Average years of practice in Jodhpur city - 9.9 years.Standard deviation is of \pm 8.8 years and Range is 0.5 to 42 years. Number of Visits $-82.2 \%$ of the dentists completed root canal treatment in 2 visits, $16.7 \%$ in 3 visits and $1.1 \%$ in 4 visits. 

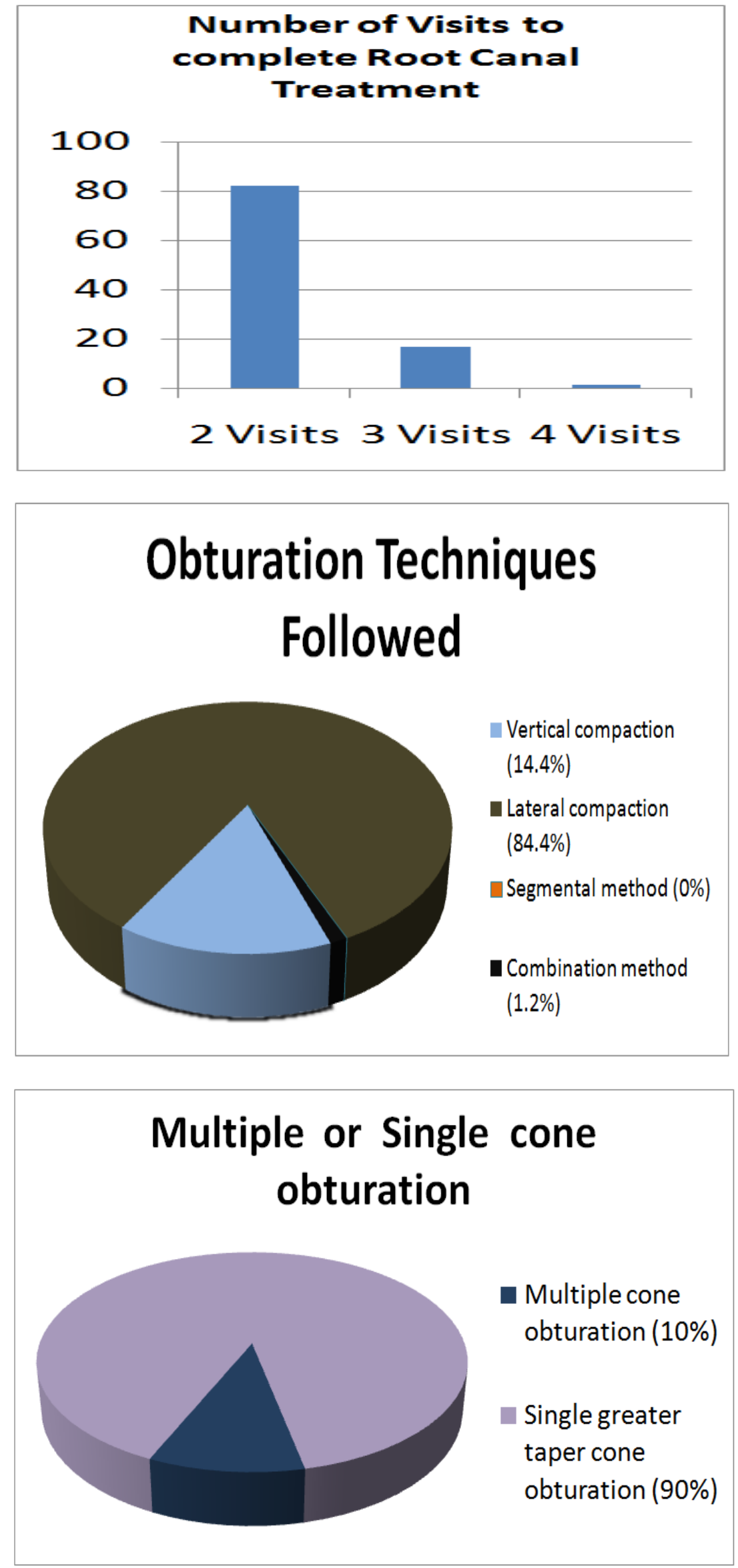

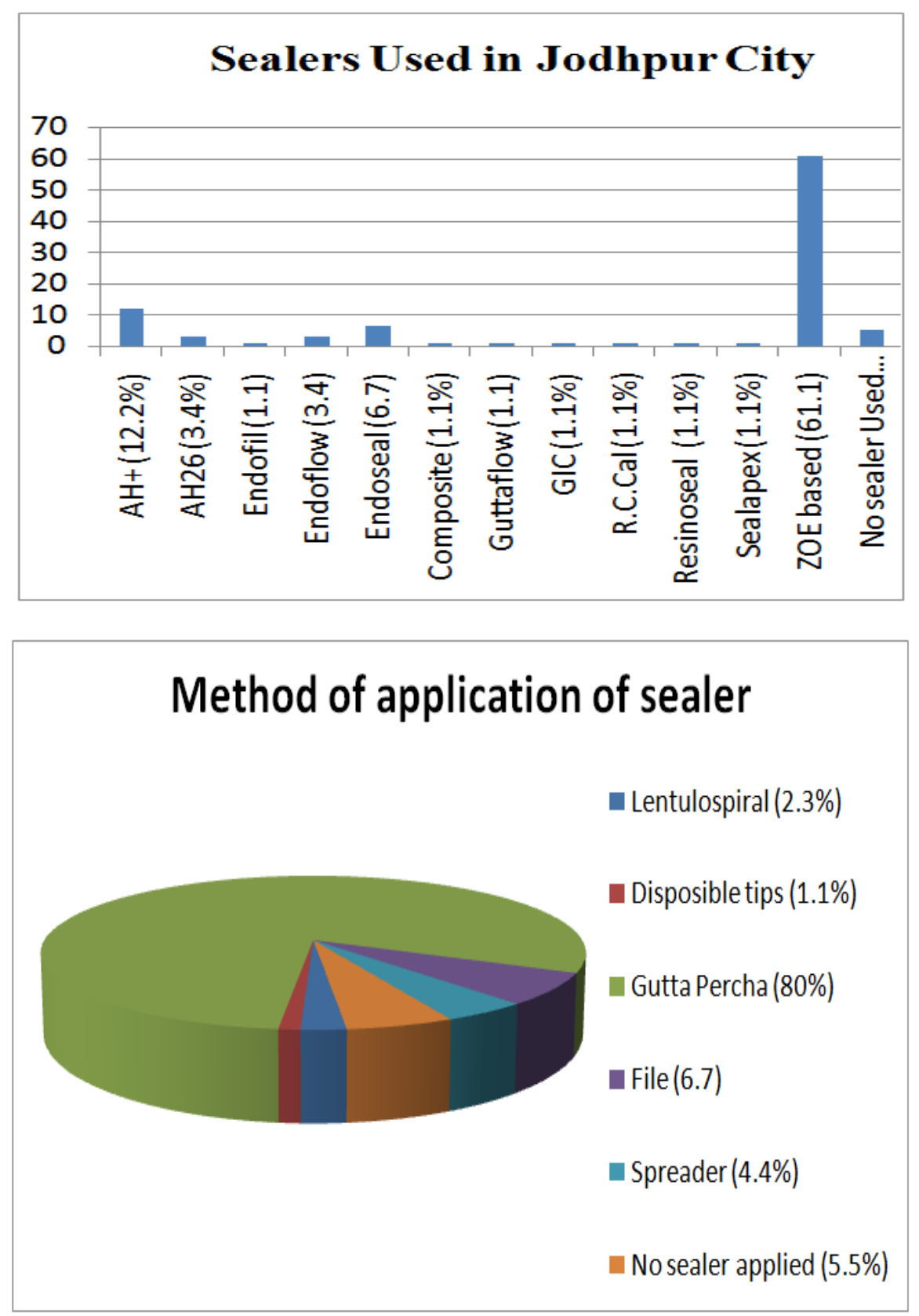

All dentists claimed to perform single visit endodontics but none specified the percentage of single vs. multiple visit endodontics.

Time between Cleaning \& Shaping and Obturation - Average time of obturation after cleaning and shaping was 3.01 days with standard deviation of \pm 0.1 .

Range -2 days to 7 days

\section{Discussion}

According to the survey of this study, $82.2 \%$ of the dentists preferred to complete the root canal treatment in just two visits irrespective of the vitality of the tooth. Average time of obturation after cleaning and shaping is 3 days with a range of 2 to 7 days. Talking in terms of techniques of obturation, lateral compaction is the most commonly followed by the vertical compaction technique and least practiced was the combination method, and segmental method was not at all practiced.Single cone technique was most widely used as much as $90 \%$ as compared to multiple cones $(10 \%)$, suggestive of useof rotary system over hand files. Most popularly used sealer was ZOE based and followed by AH+ and endoseal. 5\% of dentists did not use any sealer and obturated with the gutta percha point only which is the matter of concern. 
Endoflow and AH26 together were used by 6.8 percent ofdentists.Ideally speaking the lentulospiral is the most preferred method of application of sealer into the canal, which was followed only by $2.3 \%$ of the dentist population.Most commonly used method of sealer application was coating the gutta percha points $(80 \%)$ with sealer followed by the $\mathrm{K}$ file and spreader $6.7 \%$ and $4.4 \%$ respectively. $1.1 \%$ of dentists used dispensing tips for loading of sealers in canals.A survey done by Raoof et al 2014 in Iran to gather information on the materials and methods employed in root canal treatment by general practitioners, revealed similar results as the present study.Sealer of choice was ZOE (51\%) followed by the resin based $(44.5 \%)$ in the Iranian population, where as in Jodhpur it was $61.1 \%$ and $17.1 \%$ respectively. ${ }^{(3)}$ For the choice of obturation technique the majority $(81.7 \%)$ of participants used cold lateral condensation while vertical condensation was selected by $12 \%$ of the dentists. ${ }^{(3)}$

Another survey done in Turkey in 2012 revealed that resin based sealers were most commonly used (48\%) followed by calcium hydroxide based (35\%) root canal sealers and ZOE based sealers were not used at all in the Turkey survey contrary to Jodhpur where ZOE based sealer was the most commonly used. The majority $(66 \%)$ of the respondents in Turkey preferred cold lateral condensation as an obturation technique which was similar to that of Jodhpur. ${ }^{(1)}$ According to a survey done in Jaipur, Rajasthan either cold or warm lateral condensation of gutta percha was used by $91 \%$; other less popular options included warm vertical condensation, thermo plasticized gutta percha techniquesMajority of dentists reported the use of a zinc oxide based sealer with the gutta percha points $(55 \%)$ followed by a calcium hydroxide based sealer $(32 \%)$ and resin based sealer(13\%). Results of this survey coincide with the study conducted in Jodhpur. ${ }^{(2)}$

\section{Conclusion}

Endodontics is a dynamic, evolving discipline with considerable advances in techniques and materials over the last decade. The present small scale study may not necessarily represent the true picture, however, this study demonstrated that dentists performed procedures which often deviated from well acknowledged endodontic quality guidelines and is often in general practice carried out under less than optimal conditions.

This survey shows the importance of establishing higher specialist training or continuing dental education for practitioners to update their knowledge.

Conflict Of Interest - No potential conflict of interest relevant to this article was reported.

\section{References}

[1]. GulCelikUnal et al. Survey of attitudes, materials and methods preferred in root canal therapy by general dental practice in Turkey: Part 1. Eur J Dent. 2012;6(4):376-384

[2]. N. Mehta et al. Endodontic Trends: Where We Are And Where We Should Be-A Survey Report. People's Journal of Scientific Research, Vol. 6(2), July 2013

[3]. MaryamRaoof. PreferredMaterials and MethodsEmployed for EndodonticTreatment by Iranian General Practitioners. IranianEndodontic Journal 2015;10(2): 112-116

[4]. Quality guidelines for endodontic treatment: consensus report of the European Society of Endodontology. IntEndod J. 2006;39(12):921-30

[5]. Bjorndal L. The adoption of new endodontic technology amongst Danish general dental practitioners. IntEndod J. 2005;38(1):52-8

[6]. Lee M. et al. Current trends in endodontic practice: emergency treatments and technological armamentarium. J Endod. 2009;35(1):35-9

[7]. Khalid S. Al-Fouzan. A survey of root canal treatment of molar teethby general dental practitioners in private practicein Saudi Arabia. The Saudi Dental Journal (2010) 22, 113-117

[8]. Ravanshad S. Survey of Endodontic Practice amongst Iranian Dentists Participating Restorative Dentistry Congress in Shiraz, November 2007. Iran Endod J. 2008;2(4):135

[9]. Mohammad Ali Mozayeni et al. A Survey on NiTi Rotary Instruments Usage by Endodontists and General Dentist in Tehran.Epub 2009 Feb 7. IntEndod J 2009;42:313-21 